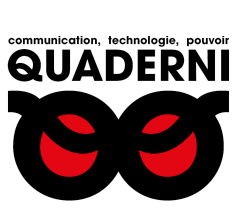

\title{
Quaderni
}

Communication, technologies, pouvoir

95 | Hiver 2017-2018

Logiques numériques des radicalisations

\section{Modéliser et expliquer les radicalisations}

Entretien avec Gérald Bonner et Serge Galam

Gérald Bronner, Serge Galam et Virginie Tournay

\section{(2) OpenEdition}

Journals

Édition électronique

URL : https://journals.openedition.org/quaderni/1143

DOI : 10.4000/quaderni.1143

ISSN : 2105-2956

Éditeur

Les éditions de la Maison des sciences de l'Homme

Édition imprimée

Date de publication : 5 février 2018

Pagination : 65-78

Référence électronique

Gérald Bronner, Serge Galam et Virginie Tournay, « Modéliser et expliquer les radicalisations »,

Quaderni [En ligne], 95 | Hiver 2017-2018, mis en ligne le 05 février 2020, consulté le 04 septembre

2021. URL : http://journals.openedition.org/quaderni/1143; DOI : https://doi.org/10.4000/quaderni. 1143 


\section{$D$ ossier}

Modéliser et expliquer les radicalisations Entretien avec Gérald Bonner et Serge Galam

Professeur de sociologie Membre de l'Académie des technologies et membre de l'Académie nationale de médecine.

Serge

Galam

Physicien

Directeur de recherche au CNRS

par Virginie Tournay

Directrice de Recherche au CNRS CEVIPOF, SciencesPo
Gérald Bronner : Je ne suis pas un spécialiste de l'islam, j'interviens comme spécialiste des croyances. L'idéologie djihadiste présente des éléments narratifs classiques dans le monde des croyances comme le millénarisme, la posture victimaire et les théories du complot, mais aussi des spécificités. C'est le propre de la sociologie de travailler sur l'hybridation entre les invariances des phénomènes sociaux et les variables.

Il y a plusieurs points à préciser sur les mécanismes et les expressions de la radicalisation aujourd'hui.

Tout d'abord, dans le rapport entre la radicalisation et le monde numérique, il ne faut pas envisager internet comme un monde seulement virtuel, même si les individus se constituent des identités fictives. Il ne s'agit pas d'un monde déconnecté du réel puisque ce sont des personnes qui interviennent dans cet univers digital. Il n'y a donc pas lieu de s'étonner que les processus de radicalisation puissent se dérouler à ce niveau.

Ensuite, toutes les formes de radicalisation qu'elles soient djihadistes, qu'elles émanent de certains groupes sectaires ou totalitaires, d'extrême gauche ou d'extrême droite, constituent des évènements de rareté statistique : par définition ils ne constituent pas la norme. Sur ce marché cognitif, la radicalisation constitue une offre intellectuelle mais il est peu aisé a priori pour les « offreurs » (les propagandistes, les recruteurs) de trouver des « acheteurs » car ce type de vision du monde implique des formes de ruptures sociales et cognitives qu'il n'est pas facile d'obtenir sans un certain nombre d'étapes préalables. 
Enfin, internet permet de créer de la fluidité dans les liens sociaux. C'est une grande différence par rapport au monde social. Par exemple, les témoins de Jéhovah qui ont une proposition intellectuelle difficile à vendre font du porte à porte. Ils vendent leur vision radicale du monde en essayant de trouver une demande, mais la perte de temps est énorme parce qu'ils cherchent à l'aveugle. Chacun essaie d'attirer du temps de cerveau disponible dans un environnement cacophonique. Internet limite les effets de cette recherche à l'aveugle ; la propagande interpelle directement les esprits qui recherchent l'héroïsme. Internet fluidifie les relations pour le meilleur et pour le pire. Cela permet à l'offre de radicalisation de trouver la demande et réciproquement. Le degré de séparation de Milgram est réduit avec les réseaux sociaux (il tombe à 4 au lieu de 6). Il est donc plus facile de trouver l'offre correspondante selon la motivation des demandeurs et des offreurs. Or, les croyants ou les radicaux sont plus motivés que les citoyens lambda. Ils ne sont pas plus représentatifs mais plus visibles que les autres. Internet offre une chambre d'écho aux propositions radicales. Elles deviennent plus virales qu'elles ne l'étaient dans le monde social conventionnel.

Serge Galam : Je ne suis pas un spécialiste du radicalisme islamiste en tant que tel. Mes travaux se focalisent sur les phénomènes d'émergence d'une nouvelle pratique en rupture avec une pratique majoritaire dans un ensemble social ou politique. Au départ extrêmement minoritaires, ces pratiques de rupture semblent toujours vouées à le rester, et dans la grande majorité des cas elles le restent. Mais parfois, après de longues périodes de stagnation, tout d'un coup, contre toute attente et par surprise, une de ses pratiques de rupture envahit toute une population pour y devenir majoritaire.

Mon approche se fonde d'abord sur l'hypothèse qu'il existe des mécanismes universels qui soustendent tous les phénomènes de propagation d'une opinion, d'une attitude ou d'une vision, et ce quels que soient leurs nature, contenu, implication, contexte. Ensuite, je postule que ces mécanismes obéissent à des lois qui peuvent être mises en équations. Cette approche s'inscrit dans le cadre de la sociophysique, nouveau domaine en émergence parmi les physiciens au niveau international, qui s'inspire des phénomènes collectifs dans la matière pour créer des modèles de comportements sociaux et politiques.

Je distingue deux niveaux. Le premier n'est pas de mon ressort et concerne le processus par lequel des individus donnés basculent par eux-mêmes dans un choix ultra minoritaire de rupture à un moment donné. C'est une démarche solitaire, personnelle dont la compréhension dépend de la spécificité du choix et relève des sciences humaines et sociales.

Ces individus sont d'une certaine façon des spécialistes de cette position extrême, qu'en général ils ont théorisée. Par souci de simplicité, nous les désignerons comme « professionnels ». À l'opposé, tous les autres individus seront désignés comme «dominants », dominant signifiant simplement ceux qui pratiquent le comportement ou choix majoritaire lorsque l'option radicale émerge. Il n'y a là aucun sousentendu de domination sur les autres, c'est juste la désignation du standard accepté et 
pratiqué par la grande majorité d'une population donnée à un moment donné.

Le deuxième niveau du phénomène de propagation minoritaire est essentiel, peu compris et intervient en parallèle du premier. Il s'agit du passage d'une réalité agrégée à une réalité collective résultant d'interactions entre les membres qui la composent. Sans interactions entre individus, nous avons un certain nombre de professionnels dispersés parmi une majorité de dominants. Additionner les uns et les autres donne une réalité agrégée pour laquelle le rapport de proportion est extrêmement défavorable aux professionnels. Ils sont inexistants socialement.

Cependant, la réalité n'est pas figée, elle évolue car ces quelques professionnels n'en sont pas moins des individus appartenant à des réseaux sociaux, des communautés, dont les membres partagent le même terreau, la même culture, les mêmes valeurs, des profils économiques semblables. Et dans chacun de ces réseaux, les gens discutent entre eux, argumentent, communiquent sur le temps qu'il fait mais aussi sur les sujets importants qui les concernent ou qui les intéressent. On est donc confronté à un ensemble dans lequel une collection d'individus qui a fait un choix de rupture, est plongée dans un ensemble bien plus grand d'individus similaires, mais qui eux pratiquent le choix en cours. Tous discutent entre eux sans discrimination ni ségrégation liées au choix radical qui, limité à un individu est sans conséquence notable.

\section{Ces échanges sont tout à fait naturels, en quoi sont-ils significatifs ?}

SG : Lors de ces échanges informels, amicaux, des professionnels vont parfois convaincre des dominants de changer de point de vue, ces derniers devenant alors des « radicaux ». Ils se distinguent des professionnels par le fait que leur " conversion » a été le fait d'échanges rationnels sur la question. Sensibles sur le choix de rupture, ils l'adoptent sans pour autant être bornés. Ils se sont fait leur opinion en discutant, la défendent, vont même essayer de convaincre d'autres dominants de les rejoindre, mais ils restent à l'écoute des contre-arguments et sont susceptibles de changer de nouveau de choix. Ce sont des flotteurs en opposition aux professionnels qui sont des inflexibles. Ces échanges génèrent une dynamique d'opinion continue, et la question centrale est de pouvoir anticiper dans quelle direction elle va se développer et dans quel rapport de force elle va éventuellement se stabiliser. Il s'agit d'anticiper si une idée controversée présente un risque de se propager au point de constituer une norme nouvelle pour les groupes sociaux, ou si au contraire elle est amenée à rester minoritaire ou à baisser.

Pour cela, la modélisation sociophysique construit une représentation simplifiée de la réalité tout en essayant d'en capturer des ingrédients décisifs. Ainsi, à partir d'hypothèses sur les interactions entre les individus dans des discussions informelles en petits groupes aléatoires, les équations obtenues révèlent une dynamique de seuil. C'est-à-dire qu'il existe des valeurs de proportion de radicaux au-dessus desquels toute fraction initiale de radicaux va 
pouvoir convertir une grande partie des dominants pour transformer le choix de rupture en nouveau choix dominant. Si cette proportion initiale est en dessous de ce seuil, les radicaux non seulement n'arriveront pas à convertir le reste de la population, mais ils renonceront pour une bonne part à leur choix radical pour redevenir dominants. Les professionnels resteront alors essentiellement isolés et sans impact notable.

Ce résultat est important parce qu'il montre qu'en présence de nouvelles pratiques controversées, si le nombre de radicaux est en-dessous du seuil, cela n'aura pas d'impact sur la collectivité et qu'il n'y a pas lieu de s'en inquiéter. Cependant, si on s'approche du seuil et qu'on le dépasse, ces pratiques mêmes ultra-minoritaires vont se propager d'un coup et plus rien ne pourra s'y opposer. Même si les pouvoirs publics parviennent alors à neutraliser les professionnels à l'origine de la pratique controversée, les inflexibles, le phénomène perdurera car les flotteurs radicalisés resteront en proportion supérieure au seuil et continueront à se propager. Le choix de rupture restera installé dans la société. C'est un élément indispensable pour comprendre pourquoi au dessus du seuil toute tentative de déradicalisation est vouée à l'échec.

\section{Comment peut-on connaître la valeur de ce seuil et de quoi dépend-t-elle?}

SG : La détermination des valeurs seuils est mathématiquement précise mais celles-ci ne doivent pas être prises « au pied du chiffre » car résultant de modèles qui ne sont pas la réalité. Cependant, quelles que soient les hypothèses d'interaction introduites dans les modèles, on est toujours bien loin des 50\% et surtout, quel que soit le sujet, le seuil est dédoublé de façon asymétrique. Il va être plus grand que $50 \%$ pour un des choix et plus petit que $50 \%$ pour le choix opposé. Cette asymétrie est un résultat robuste par rapport à la réalité des phénomènes. Mais que le seuil soit au-dessus ou en dessous de $50 \%$ pour un choix donné, n'a rien à voir avec la proportion initiale d'adhésion à ce choix. Pour un choix donné, le seuil sera d'autant plus bas que les biais cognitifs et les préjugés dominants du groupe sont en harmonie avec lui.

Par exemple dans le cas du Brexit, alors qu'avant le lancement de la campagne pour le référendum, « rester dans l'Europe » était majoritaire, son seuil était autour de $80 \%$. Dès lors que les discussions ont commencé, contre toute attente cette proportion n'a fait que décroître pour finalement passer sous la barre des 50\%. L'inverse s'est simultanément produit pour le soutien au Brexit, qui pour l'emporter, devait démarrer avec seulement plus de $20 \%$ des intentions de vote. La victoire du Brexit était donc tout à fait prédictible, du moins à la condition d'être informé de cette dynamique de seuils asymétriques. Clairement, le Premier ministre Cameron et ses conseillers de l'étaient pas.

\section{Comment expliquer l'origine de la conversion?}

SG : Les individus sont rationnels, c'est-à-dire qu'ils s'appuient sur les informations qu'ils ont en leur possession (expérience, éducation) pour effectuer un choix. Dans mon modèle, j'introduis l'hypothèse de la règle de majorité locale. Lorsque les gens discutent entre eux, chacun donne ses arguments de façon libre et ouverte. Avec cette hypothèse du choix rationnel majori- 
taire, chaque individu a un seul argument et tous les arguments se valent. Si bien que la majorité locale l'emporte au sein d'un groupe qui discute. Mais quand il y a autant d'arguments favorables que d'arguments opposés, un « doute collectif» surgit directement créé par les choix rationnels d'individus mettant en commun leurs informations et arguments. Du point de vue de ce choix rationnel enrichi, les deux options se valent. Le groupe est alors dans l'incapacité de choisir de façon rationnelle. La rationalité étant ainsi neutralisée dans un petit groupe, c'est l'inconscient, le préjugé ou le biais cognitif du groupe, qui prend le dessus pour décider du choix. Il est plus aisé de suivre la croyance collective puisque la raison ne peut pas s'y opposer et il n'est pas nécessaire de la justifier, les deux choix se valant.

\section{Peux-tu nous donner un ou deux exemples emblématiques de doute collectif ?}

SG : Une réforme dans une société ou dans une entreprise est emblématique. À force d'en discuter, on arrive souvent à des arguments qui se neutralisent, qui s'égalisent en faveur et en défaveur de la réforme. Si on demande à un individu de changer son cadre de vie en ayant autant d'avantages que d'inconvénients à le faire, il préférera garder ce qu'il a. Il ne prendra pas le risque d'aller vers une réforme où il n'a a priori pas plus d'avantages que d'inconvénients. La prime est au statu quo dans le groupe qui doute collectivement. Un autre cas de figure est l'innovation, par exemple, un groupe qui doit décider de l'achat d'un nouveau smartphone. Si autant d'avantages que d'inconvénients sont avancés entre un Androïd et un iPhone, la prime ira souvent à la nouveauté, le dernier modèle étant privilégié. Dans ces deux exemples c'est un même groupe qui dans un cas va être enclin à favoriser le statu quo et dans l'autre la nouveauté car en fonction du contenu les préjugés sont opposés.

Pour des réformes, les seuils peuvent être à $80 \%$ et $20 \%$. Il suffit donc à l'opinion défavorable d'être au-dessus de $20 \%$ pour commencer à monter alors que pour celle en faveur de la réforme, l'adhésion de départ doit être au-dessus de $80 \%$ pour augmenter. Il y a ainsi des enjeux qui sont perdus d'avance. Pour une nouvelle technologie, c'est le contraire, dès qu'il y a plus de $20 \%$ d'individus qui choisissent la nouveauté, c'est gagné pour le nouveau produit. Trois ingrédients sont importants : la valeur du seuil, rester au-dessus de ce seuil, et être en harmonie avec le biais collectif activé en cas de doute. C'est un jeu subtil. Il est parfois difficile de connaître le préjugé qui sera activé dans le cas d'un choix d'une pratique ou d'une opinion en rupture.

\section{Quels sont les mécanismes de la radica- lisation?}

SG : Dans une population donnée susceptible d'être convaincue par la forme radicale d'une certaine pratique, tant que le nombre de radicaux ne dépasse pas la valeur du seuil, la pratique modérée reste dominante. En revanche, au-dessus du seuil, l'augmentation des radicaux se fait d'abord très lentement sans que ce soit perceptible de l'extérieur et puis tout s'accélère, la majorité change de camp et personne ne comprend ce qui s'est passé. Cela fait écho aux témoignages du type : "Cela fait trente ans que nous avons une coexistence pacifique, il n'y a pas de radicalisme, 
et là depuis trois mois, sans aucun signe annonciateur, il y a des actes délétères partout avec une invasion d'islamistes ». C'est typiquement les commentaires que l'on observe dans des villes comme Lunel. Le modèle explicite clairement le processus lent d'accumulation qui ne donne rien, qui passe le seuil et se propage à une majorité. D'où l'importance d'avoir une bonne estimation de la proportion de ces professionnels. Un autre aspect important spécifique à la radicalisation concerne la lenteur du processus et l'asymétrie $\mathrm{du}$ processus. Une fois qu'un individu est radicalisé, il devient difficile pour lui de revenir en arrière. Cela peut être introduit dans le modèle avec le concept d'inflexibles. Leur proportion a un impact déterminant sur la valeur des seuils.

GB : La radicalisation obéit à une pensée incrémentielle. Le terme de la proposition radicale serait souvent inacceptable pour l'esprit en train de se radicaliser. Cette entrée progressive permet de faire passer pour raisonnable chaque marche de l'escalier du fanatisme. C'est un processus que les sectes ont parfaitement intégré en dissimulant bien souvent les aspects les plus déroutants de leur doctrine. Cette ascension vers la radicalité peut être plus ou moins rapide mais elle nécessite le plus souvent un processus progressif qui conduit l'individu à réduire la puissance de ses doutes par rapport à la doctrine. Ce processus incrémentiel affecte les normes auxquelles l'individu adhérait. Plus les minorités sont actives, plus elles peuvent créer des points de bascule, des effets non-linéaires ou non-monotones de l'opinion publique que Serge propose d'approcher par des modèles multi-agents. Il s'agit d'un phénomène connu en sciences sociales, il s'apparente au paradoxe d'Olson ou le paradoxe des minorités.
Les modèles de Serge offrent une description dudit phénomène, peut-être pas une explication ni une prédiction. Nous avons un point de désaccord à ce propos mais son travail de modélisation est très élégant en terme de description.

\section{Comment s'articule le rapport entre la science et la religion dans l'islamisme ? On a l'im- pression d'une dissonance cognitive entre la science et la maîtrise parfaite de la technologie.}

GB : Sur la maîtrise technique, on sait que les islamistes ont été très précocement sur la toile. Un islam media center a été créé en 1994. Des sites montraient comment préparer des explosifs et propager le djihadiisme. On sait que cela fonctionnait beaucoup par vidéo parce que ce sont des acteurs non autorisés sur le marché public. Daesh a publié un nombre impressionnant de vidéos plutôt bien faites avec des codes commentés. Les réponses institutionnelles sont très faibles en termes quantitatifs. Pour une vidéo de Stop-Djihadisme ${ }^{1}$, il y a cinq cents ou mille vidéos du côté djihadiste. Le nombre de comptes Twitter favorables au djihad est immense. Maintenant il circule sur d'autres réseaux plus protégés comme Telegram pour éviter l'intrusion du pouvoir politique. Tous les comptes ne sont pas forcément coordonnés. Le théoricien djihadiste Abou Moussab al-Souri a prophétisé dans les années 2000 le djihadisme en rhizome. Contrairement à Al-Qaïda qui avait une coordination verticale, les adeptes de Daesh effectuent un travail horizontal correspondant à la structure d'information sur internet. Cette organisation s'adapte relativement bien au passage à l'acte car il est évident que Daesh revendique des attentats qui n'ont pas été fomentés par eux. En plus, l'outil utilisé est facile d'accès : cela 
peut-être un marteau, un couteau, une voiture, un camion loué susceptible de faire des dizaines de morts à Nice. Donc, c'est vraiment une activité en rhizome.

Bien qu'ils soient anti-darwiniens, ils ne se revendiquent pas non scientifiques. Si les pays musulmans donnent plutôt raison à la religion qu'à la science, les entretiens avec des radicalisés sont marqués par le fait qu'ils revendiquent une sorte de pré-science dans le Coran souvent sur des points de détails, de petites découvertes scientifiques faites récemment. Cet aspect est caractéristique des textes qui se veulent prophétiques. Ils prophétisent toujours l'ultra-présent. On retrouve la même tendance chez les interprètes des textes de Nostradamus par exemple. Dans ce système de pensée, la religion n'est pas opposée à la science mais plutôt la religion précède la science sur de nombreux éléments. Quand la religion n'est pas d'accord avec la théorie de l'évolution, on trouve des arguments pseudo-scientifiques comme la théorie du dessein intelligent pour s'y opposer.

\section{Quelle est la place des réseaux sociaux dans ce mécanisme d'amplification des opinions minoritaires?}

SG : Les réseaux sociaux sont un catalyseur du phénomène mais sont sans effet sur la valeur seuil $\mathrm{du}$ basculement de l'opinion. Ce sont toujours des groupes et des individus qui réagissent mais à une échelle différente. Les réseaux sociaux brassent des populations et des distances beaucoup plus grandes même si la surface d'échange est largement définie par la circulation des discours qui recrée un entre soi dans des formes d'autoconviction. Avant internet, il fallait que les gens se rencontrent physiquement, donc le processus de conversion prenait plus de temps.

GB : Dans mon ouvrage La Démocratie des crédules publié en 2013, j'ai proposé un modèle intellectuel mobilisant quelques données empiriques largement confirmées ensuite par les sciences sociales computationnelles sur la base de données massives. Dans la vie réelle, on ne se radicalise pas tout seul à quelques exceptions près ; il y a des effets de polarisation qu'amplifie Internet. La façon dont fonctionne notre cerveau par le biais de confirmation alliée à la nature des algorithmes qui tendent à créer des chambres d'écho organise les conditions de la survie de propositions intellectuelles qui devraient pourtant disparaître de la vie sociale parce que le coût de leur endossement est très élevé. Un des processus qui permet à ces propositions intellectuelles de survivre est de faire groupe et de s'isoler. C'est pour cela que les groupes qui ont des tendances totalitaires préconisent l'isolement. On a vu certains groupes djihadistes par exemple occuper des fermes isolées. Sur internet, il y a des villages virtuels où les gens se fréquentent entre eux. Cette chambre d'écho est d'autant plus marquée que le point de vue qui y est défendu est considéré comme hostile par l'environnement social. Les clusters cognitifs sont caractéristiques y compris dans la vie physique, comme à travers les mosquées ou les prisons. On est donc en présence de marchés cognitifs fermés où se créent des lieux de polarisation. Les algorithmes autorisent et encouragent cela. Toutes ces conditions réunies font qu'il n'y a pas lieu de s'étonner qu'internet et radicalisation fonctionnent bien ensemble. 


\section{Comment interviennent les controverses sur la structuration des opinions?}

SG : L'entre-soi est marqué par le partage des mêmes croyances, des mêmes biais cognitifs. Dans un groupe modéré, l'intervention de professionnels extérieurs aura des difficultés à marquer son impact. Si un message choquant du type « on ne boit plus d'alcool » circule, la mise en doute morale de cet énoncé peut faire basculer du côté radical certains sous-groupes, notamment si cet énoncé est associé à des préceptes religieux ou s'il fait écho à des contextes culturels précis. En cas de doute, le choix ne s'établit plus sur un mode rationnel mais fait appel aux préjugés dominants du groupe. Si bien que dans les modélisations de sociophysique, on arrive à intégrer le basculement des gens qui, au départ, étaient hostiles à de tels énoncés.

Un exemple concret est l'élection de Trump. Quand il faisait des déclarations sexistes, les gens étaient choqués et plus ils étaient choqués, plus ils voulaient discuter, et plus ils voulaient discuter, plus les possibilités d'avoir ces doutes collectifs apparaissaient. La bascule du vote ne trouve pas son explication dans une désinhibition consciente. Les gens qui ont voté pour lui n'étaient pas nécessairement sexistes. Pour la plupart ils devaient même être antisexistes, mais si lors de discussions leur anti-sexisme rationnel contre Trump se trouvait contrebalancé par un rejet du système avec Clinton, les deux choix in fine se valaient : c'est leur sexisme refoulé qui a fait le choix de Trump. Si la raison consciente nous empêche souvent d'agir selon nos préjugés, quand elle ne peut plus choisir face à un doute collectif, c'est le préjugé qui va « subreptice- ment » dominer la prise de décision. Bien que rejetés, de nombreux préjugés sexistes restent intégrés dans notre inconscient.

En terme de stratégie politique, cela revient à considérer qu'un homme politique minoritaire a intérêt à provoquer une controverse si celle-ci correspond au biais cognitif susceptible d'être activé quand il s'exprimera. Sinon, il baissera dans les sondages. Au départ, les préjugés activés à propos Donald Trump jouaient contre lui, il perdait des voix dans les sondages, un des principaux préjugés étant son manque d'expérience politique. Ensuite, quand il faisait des affirmations racistes, cela le faisait baisser encore plus, mais seulement dans un premier temps, car cela activait en parallèle un nouveau préjugé qui lui était favorable et ensuite il remontait dans les sondages car il avait mis le doute de son côté. Cela contraste avec la campagne politique française qui, en dépit de son agressivité, contenait très peu d'affirmations choquantes. C'était davantage un jeu entre inflexibles qui finalement n'a pas conduit à faire basculer les modérés.

Un autre exemple concerne les recrutements, une femme a de nos jours toutes les chances d'être retenue face à un homme dès lors que son dossier est supérieur. En revanche, quand les deux dossiers se valent, le doute collectif s'installe, les biais ressortent et c'est souvent le dossier masculin qui sera privilégié sans faire explicitement appel au sexisme. C'est aussi bien l'un que l'autre, et par « hasard» c'est l'homme qui est choisi.

GB : Est-il possible d'avoir une imposition de la pensée extrême dans la société, c'est-à-dire 
un déplacement incrémentiel de la norme ? Sur ce point, je ne suis pas très boudonien, qui était plutôt évolutionniste et envisageait des crans de sécurité en matière de normes morales. Pour lui, sur des questions telles que la peine de mort, on ne peut pas revenir en arrière. Cela n'est pas si sûr. En effet, l'abolition de la peine de mort était avant-gardiste puisqu'elle a précédé le basculement de l'opinion publique. Aujourd'hui, les sondages récents montrent qu'il y a un recul de l'opinion sur cette question. Comme un bon popperien, je mise sur le caractère imprédictible de l'histoire humaine. Cela n'empêche sans doute pas d'observer des évolutions majeures dans notre histoire commune. On peut l'expliquer par le fait que certaines propositions intellectuelles ont une charge universaliste plus importantes que les autres. C'est pourquoi les revendications d'égalité (homosexuelle, féminisme) peuvent atteindre plus facilement une norme partagée que d'autres propositions qui sont moins transubjectives et qui ont une charge universaliste moins forte. Il me paraît difficile de remettre en cause la condamnation de l'esclavage. C'est toujours une possibilité dans certains contextes précis mais cela est moins probable. La charge universaliste affaiblit ces probabilités parce que la démonstration selon laquelle un homme est supérieur à un autre est très difficile à faire. Si la force physique est considérée comme un critère déterminant alors les hommes sont supérieurs aux femmes et les gorilles seraient supérieurs aux êtres humains ! Donc ce qui permet d'évaluer les êtres humains, c'est leur système nerveux et de ce point de vue, les femmes sont l'égal des hommes. Il y a des débats sérieux sur les différences cognitives hommes-femmes. Cela se défend tout à fait mais par contre, ce qui ne se défend pas - même s'il y a des spécificités cognitives - c'est l'idée d'une supériorité. La proposition égalité homme femme détient une charge universaliste objective rationnelle, de la même façon que la terre n'est pas plate. La revendication d'une supériorité essentielle ou de celle des « platistes » peut être fractionnée dans de multiples identités qui nuisent à l'universalisme. Il n'y a pas de meilleur argument que l'égalité qui est un invariant moral susceptible de s'hybrider avec des variables. Je ne crois pas aux modèles déterministes mais à l'imprédictibilité.

\section{Les outils institutionnels à notre disposition sont-ils suffisants pour combattre la radica- lisation?}

SG : La notion de préjugé fait référence à un inconscient partagé par un groupe social qui n'est pas nécessairement activé au niveau de la conscience individuelle. C'est seulement lorsqu'il y a une équivalence entre de multiples choix rationnels que les préjugés ressortent inconsciemment dans la prise de décision collective. C'est ce phénomène qui biaise la dynamique de conviction, et malheureusement les outils institutionnels que nous avons ne permettent pas d'éradiquer des préjugés ou de modifier des biais cognitifs pour fabriquer des contre-arènes de radicalisation. On peut tout au plus les rendre indicibles. Comme pour le sexisme, ils sont insuffisants.

Une lutte contre le radicalisme purement institutionnelle est donc vouée à l'échec même si elle peut convenir à certains moments précis. Dès que le contrôle est relâché, la radicalisation repartira. Ce sont nos visions de la contre-radicalisation 
qui doivent être retravaillées. Ce devrait être des « professionnels » de la pratique modérée qui devraient entrer en jeu pour convaincre les radicaux de redevenir modérés et les modérés de le rester. La seule façon de combattre la radicalisation est de créer une dynamique symétrique au sein de groupes d'individus défendant une position inverse.

Alors que les centres de radicalisation veulent convaincre des professionnels de ne plus l'être avec des chances de succès bien minces, cela n'aura de toutes façons pas d'impact décisif au niveau collectif où tout se joue dans les échanges informels entre individus in visu et sur les réseaux sociaux. Sans avoir des « professionnels » de la modération, ces échanges resteront imperméables à la déradicalisation au profit de la seule radicalisation.

Les pouvoirs publics peuvent mener une campagne de répression énorme, mais cela repartira quand même sans des modérés qui disent «nous aussi on veut être des professionnels mais de la modération ». Comment créer une telle hybridation? Par définition un modéré n'étant pas un activiste de la conviction, le défi est d'arriveràrendre actifs des individus d'une société susceptible d'être submergée par un radicalisme pour maintenir le statut de leur société. Dire, «je ne soutiens pas telle vision mais je ne m'implique pas contre » n'a aucun impact sur la dynamique de radicalisation.

Faudrait-il donc des mécanismes institutionnels qui permettraient de créer des espaces informels dans lesquels les modérés pourraient s'exprimer et convaincre les autres?
SG : Les dynamiques de changement d'opinion ont lieu lors de rencontres dans les diners, les cafés, les prières... Dès qu'on rentre dans un cadre un peu formalisé, ce ne sont plus les mêmes types de rapport. On peut lutter contre la mafia avec de gros moyens mais on n'a jamais pu l'éradiquer en Italie. Elle se maintient et prospère car au niveau des mécanismes d'échanges informels entre les individus, aucune autre opposition ne peut être créée sur son terrain. Le terrorisme, la mafia, les différents groupes undergrounds répondent de ce même mécanisme.

On retrouve cette constante dans l'échec criant des discours anti-conspirationnistes émanant d'institutions, d'universitaires et de scientifiques car les propos n'empruntent pas les mêmes canaux que les discours conspirationnistes. La défiance est continument alimentée car les oppositions ne se situent jamais au même niveau de conviction ou d'élaboration. Au bout d'un certain temps, le débat sur un sujet controversé s'arrête mais les individus qui ont basculé dans la croyance continuent à y croire après l'arrêt de la propagation de la rumeur. Il n'y a pas de contreconspiration continue en réponse au conspirationnisme. Cette asymétrie de propagation entre pairs est très importante. Les conspirationnistes sont très actifs et restent dans un discours de conviction en s'auto-entretenant sur des réseaux sociaux spécialisés.

Après le 11 septembre 201, la rumeur d'un coup monté obtient une adhésion énorme ! Ensuite elle se stabilise car une personne convaincue ne va pas renoncer à sa croyance. Il n'y a pas ou très peu de régression possible. Tous les convaincus de l'époque le sont pour la 
plupart encore aujourd'hui. Ouvrir un débat n'est pas nécessairement favorable. Même en présence d'une immense majorité de gens en désaccord avec le discours conspirationniste, si l'opinion est en résonance avec le préjugé qui s'active en cas de doute collectif, le débat est perdu.

C'est la même chose pour les OGM ou les antennes paraboliques. À ce jour il n'y a aucune preuve scientifique que ces dernières créent des cancers, mais pour leurs détracteurs, même si on ne l'a pas encore pas prouvé, on le prouvera demain. Comme il est impossible de prouver l'inexistence de ce qui n'existe pas, le débat est perdu d'avance. Il ne faut donc pas l'initier puisque tant que les personnes opposées aux antennes paraboliques restent confinées au dessous du seuil, ils n'auront pas d'impact majeur pour la question.

GB : Dernièrement, une thèse a été proposée en Tunisie (mais non soutenue par refus des pré-rapporteurs) montrant que la Terre est plate, avec un soubassement religieux. C'est une forme de relativisme. Les radicaux profitent toujours de l'affaiblissement de la norme du vrai. C'est pourquoi les rationalistes ne doivent pas mettre leur drapeau dans leur poche. Concernant les théories de l'évolution, il ne faut pas accepter la logique qui proposerait d'enseigner les deux théories (évolution vs créationnisme). Les programmes américains (school board) sont déterminés par l'établissement et par les parents ${ }^{2}$. Le problème est l'insertion de la démocratie dans la connaissance. C'est le juridique qui tranche. On peut craindre en France beaucoup d'intimidation morale à l'université. Défendre l'universalisme et la science ce n'est pas une activité politique à proprement parler, c'est l'expression de l'éthos de la science tout simplement.

L'Éducation nationale est fondamentale. Il y a une révolution pédagogique à faire face à cette révolution de l'information et on ne part pas de rien. Plus de 150 biais cognitifs ont été découverts. Il faut se servir de cette connaissance et l'utiliser à des fins pédagogiques. Il existe par exemple des propositions intellectuelles contreintuitives comme la théorie de l'évolution. Il faut non seulement enseigner cette théorie fermement, mais aussi enseigner aux enfants pourquoi cette théorie leur résiste intellectuellement. C'est une façon d'apprivoiser le cerveau, c'est-à-dire l'esprit critique. L'accès à la connaissance n'est pas quelque chose d'intuitif. Il faut expliquer aux enfants le fonctionnement de leur cerveau. Cela peut-être fait en histoire, en mathématique, en physique et dans toutes les disciplines. On peut partir d'éléments simples comme la confusion entre la causalité et la corrélation, du raisonnement cui prodest (à qui profite le crime ?).

Je ne suis pas en faveur d'une trop forte régulation et je plaide pour des interventions non-liberticides. Je crois beaucoup à la théorie du Nudge, à l'architecture du choix, à la possibilité d'injecter des propositions intellectuelles alternatives. La démocratie doit se défendre vigoureusement. Il faut apporter de l'information aux lieux où il $\mathrm{y} \mathrm{a}$ une porosité entre les radicaux et les indécis. Qui porte cette information? Cela ne doit pas être les institutions publiques ou le pouvoir politique. Il faut réinjecter une nouvelle forme de militance, il $y$ a pleins de jeunes qui y travaillent. Par exemple, 
la zététique, certaines chaines YouTube de diffusion de la connaissance comme La tronche en biais, Hygiène mentale etc., sont des entreprises formidables.

\section{Peut-on distinguer les formes de radicalisa- tion politique et religieuse ? Y a t-il certaines formes de radicalisation dont on peut penser que le risque de basculement est probable ?}

SG : Alors que les règles de diffusion et de propagation des opinions minoritaires sont les mêmes, concernant la question religieuse deux différences sont à prendre en compte. Tout d'abord, l'inertie individuelle du changement de religion et ensuite, le choix de la religion n'est pas l'objet d'un débat public. Les discussions ont une forme publique dans les populations où certains essaient de radicaliser les autres, mais on ne discute pas s'il faut être catholique, juif, musulman ou bouddhiste. Il n'y a pas de débat public sur le choix de la religion qu'il conviendrait d'adopter sur la base d'argumentaires rationnels. Les gens dans leur ensemble n'en discutent pas.

Mais par rapport à la politique, il y a beaucoup plus d'individus flexibles que sur le plan religieux. Globalement, les individus ne changent pas facilement de religion alors que bon nombre de gens sont moins inflexibles en politique. De même, les nouvelles religions n'apparaissent pas comme de nouveaux partis politiques. Si bien que les échelles de temps sont différentes. L'absence de débats, l'inertie des positionnements et l'absence de renouvellement d'offre religieuse constituent des variables à prendre en compte plus fortement dans un contexte de radicalisation religieuse. Ces paramètres rendent le phénomène de propagation plus dangereux, souterrain, et irréversible sur la longue durée.

GB : Il y a des thèmes dans l'histoire des faits sociaux qui peuvent impliquer de la violence telle que le suicide collectif, le millénarisme ou la promesse post-mortem que l'on retrouve dans le djihadisme. Cette proposition intellectuelle suggère de tout sacrifier au profit d'une récompense, soit dans l'autre monde de la vie, soit dans le monde de maintenant. Heureusement, tous les groupes millénaristes ne produisent pas de la violence. Ce millénarisme peut être de type religieux, il peut être de type politique. Il s'agit souvent d'accélérer la fin de l'histoire (socialisme, barbarisme). L'idéologie politique peut inciter à cela. Quand il y a une touche religieuse, il y a un élément transcendantal particulièrement dangereux. On avait aussi des éléments millénaristes dans le nazisme.

D'une façon générale, la diffusion d'éléments pouvant apparaître comme des signaux faibles tels que la contestation de la théorie de l'évolution dans les écoles est inquiétante. Cela peut apparaître comme un positionnement religieux que cette thèse soit interdite au moins de 18 ans en Turquie. Aussi, l'enquête de Sébastien Roché montre que la théorie de l'évolution est contestée par plus $60 \%$ des lycéens qui se disent musulmans ${ }^{3}$. C'est inquiétant. Je suis en contact avec des chercheurs et des opérateurs de la décision publique qui travaillent sur ces questions, on a des signaux qui ne sont pas rassurants dans certains quartiers. Il suffit de voir certaines affiliations dans les écoles confessionnelles qui ont doublé. Ce qui se passe dans les prisons est apparemment effrayant. Cela ne signifie pas que 
ces idéologies vont se diffuser dans l'ensemble de la population française mais par effet de dialectique, cette visibilité de la radicalisation dans certains quartiers peut prendre d'autres formes de radicalisation, notamment à l'extrêmedroite. Tant que nous ne donnerons pas de la voix sur tous les sujets, science, vaccins, populisme etc., les radicaux gagnent du terrain. Il faut être violemment démocrate. La démocratie a le droit de se défendre.

\section{Gérald Bronner}

- The Future of Collective Beliefs, Oxford, Bardwell Press, 2011.

- La démocratie des crédules, Paris, Puf, 2013.

- « Métamorphose du croire radical», Miviludes.

Rapport au Premier ministre 2013-2014, La documentation française, 2014, p. 21-45.

- La pensée extrême, Paris, Puf, 2016.

- « À la recherche de nouvelles traces sociales» (avec David P. et Del Buono L.), Revue Européenne des sciences sociales, 1, 2018.

\section{Serge Galam}

- "Sociophysics: A Physicist's Modeling of Psycho-political Phenomena", Springer, New York (2012).

- "The Trump phenomenon: An explanation from sociophysics”, International Journal of Modern Physics B 31, 1742015 (2017).

- "Modeling Radicalization Phenomena in Heterogeneous Populations", (avec M. A. Javaronne), PLoS ONE 11(5): e0155407 (2016).

- "Stubbornness as an unfortunate key to win a public debate: an illustration from sociophysics", Mind Soc 15, 117-130 (2015).

- "Collective beliefs versus individual inflexibility: The unavoidable biases of a public debate", Physica A 390 (2011) 3036-3054.

- "The role of inflexible minorities in the breaking of democratic opinion dynamics", (avec F. Jacobs), Physica A 381 (2007) 366-376. 
$\mathrm{N} \cdot \mathrm{O} \cdot \mathrm{T} \cdot \mathrm{E} \cdot \mathrm{S}$

1. Initiative du gouvernement de Manuel Valls pour lutter contre la radicalisation islamiste, et toujours actif aujourd'hui. : < http://www.stop-djihadisme.gouv.fr/> 2. Dans la mesure où il s'agit de positions électives à l'échelon du comté, on ne saurait s'étonner de l'activisme déployé par les évangélistes depuis les années 1990 pour les conquérir et ainsi promouvoir leur lecture littérale de la bible en ce qui concerne l'origine de la Terre ou la question de l'avortement. 3. Sébastian Roché (coordinateur), Les adolescents et la loi : un état des lieux pour comprendre les relations entre jeunes et institutions publiques, volet français du programme UPYC (Understanding and Preventing Youth Crime), février 2016. 